\title{
Biocompatibility of Mg-Nd-Zn-Zr alloy with rabbit blood
}

\author{
WANG YongPing ${ }^{1,2 \dagger}$, OUYANG YuanMing $^{2 \dagger}, \mathrm{HE} \mathrm{YaoHua}^{2}$, CHEN DaoYun ${ }^{2}, \mathrm{JIANG}$ Yao $^{2 *}$, \\ MAO Lin $^{3}$, NIU JiaLin ${ }^{3}$, ZHANG Jian ${ }^{3} \&$ YUAN GuangYin ${ }^{3 *}$ \\ ${ }^{1}$ Department of Orthopaedics, First Hospital of Lanzhou University, Lanzhou 730000, China; \\ ${ }^{2}$ Department of Orthopaedics, Sixth People's Hospital of Shanghai Jiao Tong University, Shanghai 200233, China; \\ ${ }^{3}$ National Engineering Research Center of Light Alloys Net Forming, School of Materials Science and Engineering, Shanghai Jiao Tong \\ University, Shanghai 200240, China
}

Received November 29, 2012; accepted February 19, 2013; published online June 25, 2013

In this study, in vitro blood biocompatibility of Mg-Nd-Zn-Zr (JDBM) alloy was investigated to determine its suitability as a degradable medical biomaterial. Blood biocompatibility was assessed by blood cell aggregation, platelet adhesion, and protein adsorption. A titanium alloy was used as control. The results showed that the JDBM alloy did not induce significant blood cell aggregation, platelet adhesion, and protein adsorption comparison with the titanium alloy $(P>0.05)$. Our data indicate that the JDBM alloy has excellent in vitro blood compatibility, and thus can be considered as a potential degradable biomaterial for medical applications with respect to hemocompatibility.

magnesium alloy, hemocompatibility, blood cell aggregation, platelet adhesion, protein adsorption

Citation: Wang Y P, Ouyang Y M, He Y H, et al. Biocompatibility of Mg-Nd-Zn-Zr alloy with rabbit blood. Chin Sci Bull, 2013, 58: 2903-2908, doi: 10.1007/ s11434-013-5883-2

In recent years, there has been renewed interest in magnesium alloys, which are easily corroded in solutions, especially in the presence of chloride ions. Indeed, magnesium alloys have become promising degradable biomaterials [1-6]. Recent studies have revealed that magnesium alloys have good biocompatibility, may promote osteocyte growth, and may induce production of osteoblasts and osteocytes [7-10]. Owing to suitable mechanical properties and good biocompatibility, degradable magnesium alloys can be utilized in many clinical situations, such as bone repair and tissue engineering scaffolds; however, the rapid rate of corrosion and the appearance of gas bubbles in the physiologic environment impose severe limitations which have prevented further clinical applications. Hence, to utilize a magnesium alloy for clinical implantation, corrosion resistance must be improved.

Alloying, an effective and convenient method, has

$†$ These authors contributed equally to this work.

*Corresponding authors (email: jiangyao195106@163.com; gyyuan@ sjtu.edu.cn) emerged as a strategy to enhance the corrosion resistance of magnesium [11]. To improve corrosion resistance and biocompatibility of magnesium, a novel patent magnesium alloy (Mg-3Nd-0.2Zn-0.4Zr; wt.\%, denoted Shanghai JiaoDa BioMg alloy, abbreviated as JDBM) has been designed in our laboratory by the addition of neodymium, zinc, and zirconium to magnesium [12]. In a previous study, we focused on the microstructure [12] and biological properties of the JDBM alloy, revealing that the JDBM alloy has good biocompatibility in vitro [13]. However, the blood compatibility of the JDBM alloy has not been systematically studied in vitro and in vivo.

In the current study we focused on the in vitro blood compatibility of the JDBM alloy, and characterized the in vitro blood biocompatibility of the JDBM alloy because the degradation products of the JDBM alloy are absorbed into the bloodstream during the degradation process. It is expected that this work may help to confirm the in vitro blood biocompatibility of the JDBM alloy and obtain a new promising degradable magnesium alloy. 


\section{Materials and methods}

\subsection{Materials}

The JDBM alloy was obtained from the National Engineering Research Center of Light Alloy Net Forming, Shanghai Jiao Tong University. Titanium alloy (Ti-6Al-4V (control)), which is usually used in orthopedics and has good biocompatibility, was obtained from Shanghai Puwei Medical Instrument Factory Co., Ltd. (Shanghai, China). The composition of the JDBM and titanium alloys is listed in Tables 1 and 2 , respectively.

The JDBM alloy was prepared in a mild steel crucible under the protection of a mixed gas consisting of $\mathrm{SF}_{6}$ and $\mathrm{CO}_{2}$. Pure $\mathrm{Mg}(\geqslant 99.99 \%)$ was melted in the steel crucible, with pure Zn $(\geqslant 99.995 \%)$ added at a temperature of 700 $710^{\circ} \mathrm{C}$, then $\mathrm{Nd}, \mathrm{Zr}$ was added as $\mathrm{Mg}-25 \mathrm{Nd}$ and $\mathrm{Mg}-30 \mathrm{Zr}$ master alloys at a temperature of $740-760^{\circ} \mathrm{C}$ [12].

The JDBM and titanium alloys were formed into discs (10 $\mathrm{mm}$ in diameter and $2 \mathrm{~mm}$ height); the discs were ground with abrasive paper, polished with diamond abrasive paste, followed by ultrasound washing in ethanol for $10 \mathrm{~min}$, and sterilizing with ethylene oxide.

New Zealand white rabbits, clean grade and weighing $(2.5 \pm 0.3) \mathrm{kg}$, were supplied by the Shanghai Jiesijie Lab Animal Co., Ltd. (Shanghai, China; license No. SCXK [hu] 2010-0026). All experimental animals were treated humanely in accordance with the Guidance Suggestions for the Care and Use of Laboratory Animals issued by the Ministry of Science and Technology of the People's Republic of China (2006-09-30).

The study was approved by the Ethics Committee of the Sixth People's Hospital of Shanghai Jiao Tong University.

\subsection{Microstructure of JDBM alloy}

The microstructure of the JDBM alloy was characterized using a metallurgical microscope (4XI; Shanghai Precise Science Instrument Company, Shanghai, China). The morphology of the JDBM alloy was characterized using scanning electron microscopy (SEM, Hitachi S-4800, Japan).

\subsection{Blood cell aggregation}

Aggregation of blood cells, including red blood corpuscles (RBCs), white blood corpuscles (WBCs), and platelets, was evaluated using the blood cell count method. Arterial blood (3 mL) from a healthy New Zealand white rabbit was col- lected into a conical tube that contained $0.5 \mathrm{~mL}$ of potassium oxalate $(20 \mathrm{~g} / \mathrm{L})$ anticoagulant. Then, the JDBM alloy disc (test group) and titanium alloy disc (control group) were immediately immersed in blood. The number of RBCs, WBCs, and platelets were measured using an automatic hematology analyzer (Sysmex, XT-1800i, Japan) before and $8 \mathrm{~min}$ after the disc was immersed in blood [14]. Six parallel samples were evaluated in each group.

\subsection{Platelet adhesion}

Arterial blood (8 mL) was obtained from a healthy New Zealand white rabbit. Platelet-rich plasma (PRP) was generated by centrifuging the fresh blood at a rate of $2500 \mathrm{r} / \mathrm{min}$ for $5 \mathrm{~min}$, and PRP was collected into a new tube. The PRP was overlaid atop the JDBM and titanium alloy discs and incubated at $37^{\circ} \mathrm{C}$ for $1 \mathrm{~h}$. The discs were rinsed with phosphate buffer solution to remove the non-adherent platelets. The adherent platelets were fixed using $2.5 \%$ glutaraldehyde for $1 \mathrm{~h}$ at room temperature, then dehydrated with a gradient immersion in 50\%, 60\%, 70\%, 80\%, 90\%, and $100 \%$ ethanol for $10 \mathrm{~min}$ each. The discs were subjected to critical point drying and gold sputtering. Then, the surface of the JDBM and titanium alloy disc-attached platelets were observed by SEM. Five different fields were randomly counted and the values are expressed as the average number of adherent platelets per disc [15]. Six parallel samples were evaluated in each group.

\subsection{Protein adsorption}

Arterial blood ( $3 \mathrm{~mL}$ ) from a healthy New Zealand white rabbit was collected into a conical tube that contained 0.5 $\mathrm{mL}$ of potassium oxalate $(20 \mathrm{~g} / \mathrm{L})$ anticoagulant. Then, the JDBM alloy disc (test group) and titanium alloy disc (control group) were immediately immersed in blood. The total protein of plasma was measured using an automatic blood biochemistry analyzer (Hitachi 7600-020, Japan) before and $8 \mathrm{~min}$ after the disc was immersed in blood [14]. Six parallel samples were evaluated in each group.

\subsection{Statistic analysis}

Statistical analysis was performed with SPSS 13.0 (SPSS, Inc., Chicago, IL, USA) to evaluate the differences in each group; all data were expressed as the mean $\pm \mathrm{SD}$. The experimental values were analyzed using a Student's $t$-test. The statistical significance was defined as a $P<0.05$.

Table 1 Chemical composition of the JDBM alloy

\begin{tabular}{|c|c|c|c|c|c|c|c|c|}
\hline \multirow{2}{*}{ Materials } & \multicolumn{8}{|c|}{ Chemical composition (wt.\%) } \\
\hline & $\mathrm{Mg}$ & $\mathrm{Nd}$ & $\mathrm{Zn}$ & $\mathrm{Zr}$ & $\mathrm{Si}$ & $\mathrm{Ni}$ & $\mathrm{Cu}$ & $\mathrm{Fe}$ \\
\hline JDBM alloy & Balance & 2.83 & 0.20 & 0.44 & 0.018 & 0.0025 & 0.0195 & 0.0057 \\
\hline
\end{tabular}


Table 2 Chemical composition of the titanium alloy

\begin{tabular}{|c|c|c|c|c|c|c|c|c|}
\hline \multirow{2}{*}{ Materials } & \multicolumn{8}{|c|}{ Chemical composition (wt.\%) } \\
\hline & $\mathrm{Ti}$ & $\mathrm{Al}$ & $\mathrm{V}$ & $\mathrm{Fe}$ & $\mathrm{N}$ & $\mathrm{O}$ & $\mathrm{C}$ & $\mathrm{H}$ \\
\hline Titanium alloy & Balance & 6.05 & 4.13 & 0.064 & 0.012 & 0.10 & 0.01 & 0.0037 \\
\hline
\end{tabular}

\section{Results}

\subsection{Microstructure and morphology of the JDBM alloy}

As shown in Figure 1(c), a typical metallurgical microstructure of the JDBM alloy, the grain boundaries were barely recognizable due to the more homogeneous microstructure of the JDBM alloy. Therefore, alloying can remarkably change the microstructure of the JDBM alloy, which could improve the corrosion resistance and cause a different degradation behavior. The SEM morphology of the JDBM alloy in Figure 1(d) illustrates the uniform distribution of all particulates within the JDBM alloy.

\subsection{Blood cell aggregation}

The aggregation of blood cells on the surfaces of the JDBM and titanium alloy samples is shown in Figures 2-4. As shown in Figure 2(a), the reduction in RBC aggregation to the JDBM and titanium alloys was $19.9 \%$ and $29.2 \%$, respectively. There was no significant RBC aggregation on the JDBM alloy compared with the titanium alloy $(P>0.05)$. The reduction in WBC aggregation to the JDBM and titanium alloys was $31.21 \%$ and $27.8 \%$, respectively (Figure $3(\mathrm{a})$ ). There was no significant aggregation of WBCs occurring in the test group compared with the control group $(P>0.05)$. As shown in Figure 4(a), the reduction in platelet aggregation to the JDBM and titanium alloys was $19.0 \%$ and $18.9 \%$, respectively. There was no significant platelet aggregation to the JDBM alloy compared with the titanium alloy $(P>0.05)$. These results indicate that blood cells suspended in the JDBM alloy did not aggregate due to the

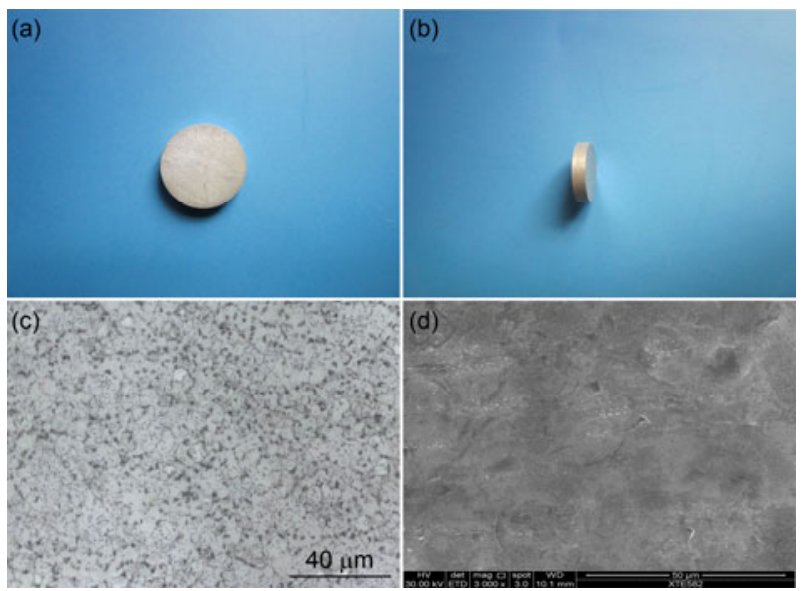

Figure 1 (a), (b) Photographs of the JDBM alloy disc. (c) Metallurgical microstructure and (d) SEM morphology of the JDBM alloy. (a)

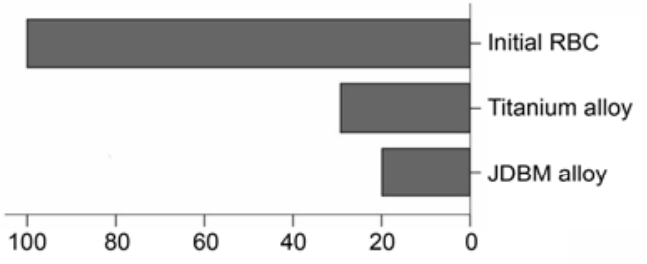

Reduction in RBC aggregation (\% of initial RBC)

(b)

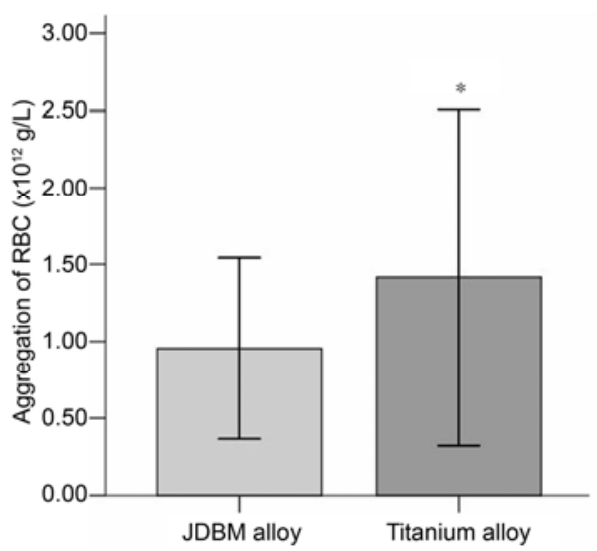

Figure 2 (a) The reduction in RBC aggregation to the JDBM alloy and titanium alloy. (b) Comparison of aggregation of RBCs on the surface of the JDBM alloy and titanium alloy $(* P>0.05)$.

(a)

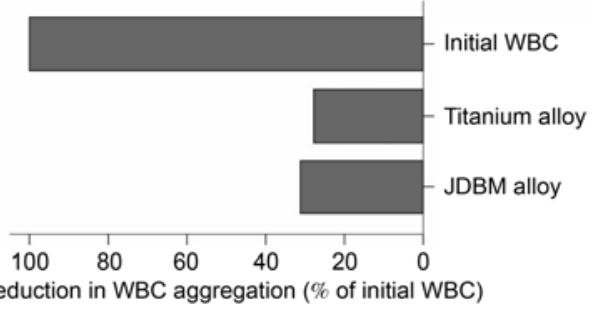

(b)

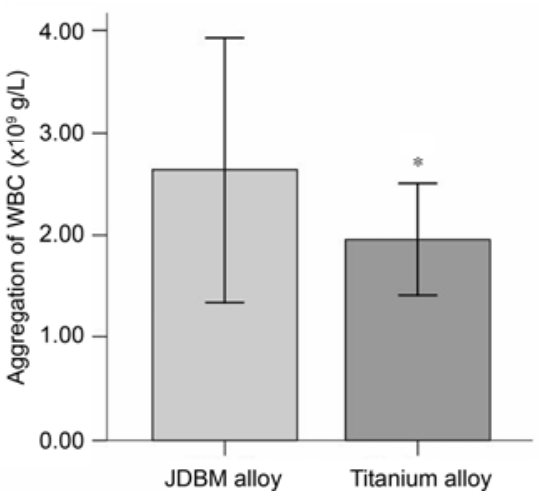

Figure 3 (a) The reduction in WBC aggregation to the JDBM alloy and titanium alloy. (b) Comparison of aggregation of WBCs on the surface of the JDBM alloy and titanium alloy $(* P>0.05)$. 
(a)
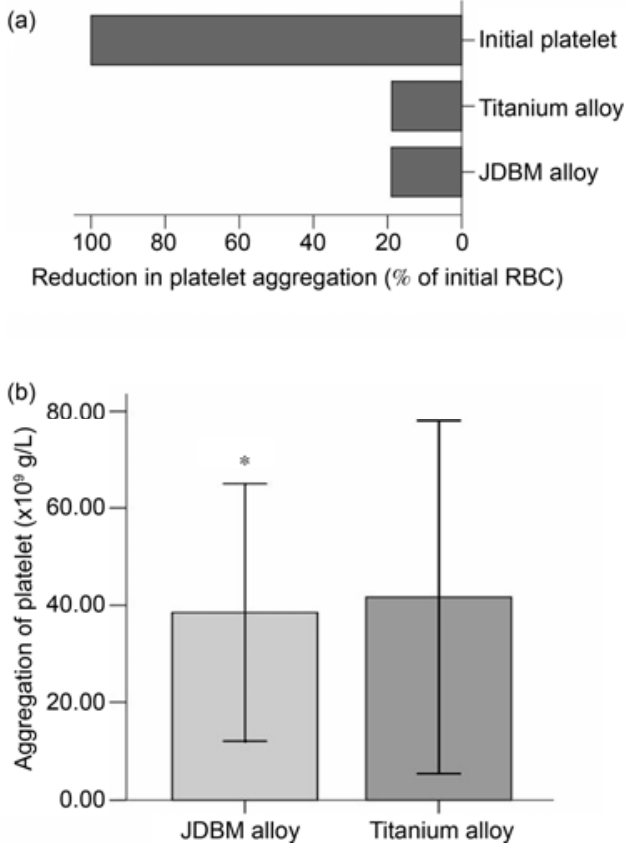

Figure 4 (a) The reduction in platelet aggregation to the JDBM and titanium alloys. (b) Comparison of aggregation of platelets on the surfaces of the JDBM and titanium alloys $(* P>0.05)$.

excellent blood compatible behavior.

\subsection{Platelet adhesion}

The platelets adherent to the JDBM and titanium alloy discs were observed via SEM. There was a small quantity of platelets adherent to the surfaces of the JDBM and titanium alloy discs. The morphologies of adherent platelets were characterized, which showed that platelets were nearly round in the test group with only one or two short pseudopodia spreading on the surface of the titanium alloy discs (Figure 5). Figure 6 illustrates the number of platelets adherent to the JDBM and titanium alloy discs after incubation in PRP for $1 \mathrm{~h}$. There was no significant platelet adhesion to the JDBM alloy compared with the titanium alloy $(P>0.05)$.

\subsection{Protein adsorption}

As shown in Figure 7(a), the reduction in protein adsorption

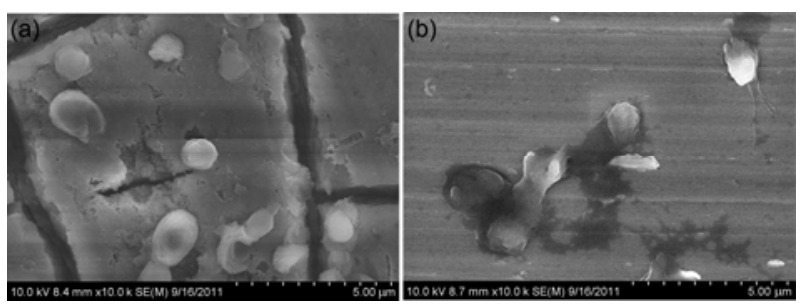

Figure 5 SEM images showing platelet adhesion on the surface of (a) the JDBM and (b) titanium alloy samples.

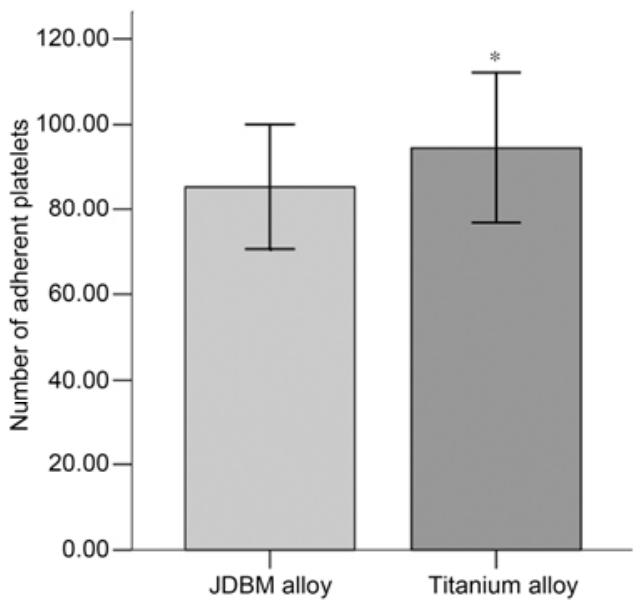

Figure 6 Comparison of platelet adhesion on the surface of the JDBM and titanium alloy samples $(* P>0.05)$.
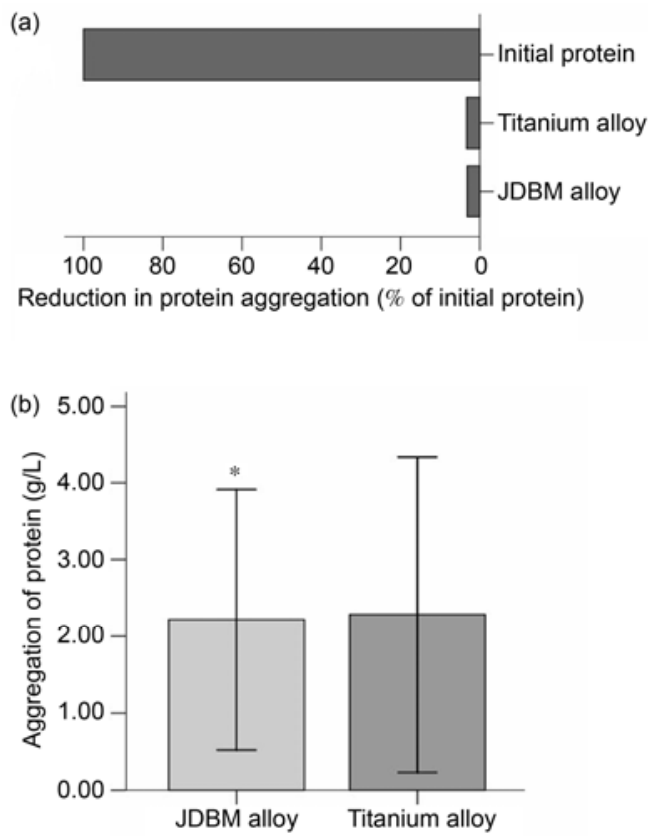

Figure 7 Comparison of protein adsorption on the surface of (a) the JDBM and (b) titanium alloy samples $\left({ }^{*} P>0.05\right)$.

to the JDBM and titanium alloys was $3.19 \%$ and $3.32 \%$, respectively. There was no significant change in total protein before and after the disc immersed in blood for $8 \mathrm{~min}$ in the test and control groups, indicating no significant adsorption of protein occurring in the test group compared with the control group $(P>0.05)$.

\section{Discussion}

Materials used for medical device fabrication must have good biocompatibility because the materials will directly contact tissues and cells after implantation in the human 
body. Specifically, the blood compatibility of these materials is a crucial factor because that contact between materials and blood causes blood cell activation and adhesion, followed by blood coagulation. Concurrently, the activated blood cells release inflammatory cytokines, resulting in damage of the local tissues and cells. As a new degradable medical biomaterial, it is important to evaluate the blood biocompatibility of the JDBM alloy from experimental studies prior to clinical application because the degradation products are absorbed into the bloodstream when the JDBM alloy is degrading in the human body. Blood is a complex living system. The response of blood to a material is determined by the following three primary factors: state of the blood; flow velocity; and the surface [16]. According to an expanded, practical definition of blood compatibility [17], a material can be evaluated using the effects of the material on erythrocytes, leukocytes, and platelets. If a material does not damage blood components upon contact, the material is defined as a blood-compatible material [18].

In the current study we focused on the blood compatibility of the JDBM alloy when used as a medical implant. Most importantly, the blood cell aggregation studies were carried out to confirm the blood compatibility of the JDBM alloy. The results showed that the reduction in RBC aggregation to the JDBM and titanium alloys was $19.9 \%$ and $29.2 \%$, respectively. There was no significant RBC aggregation to the JDBM alloy compared with the titanium alloy $(P>0.05)$. The reduction in WBC aggregation to the JDBM and titanium alloys was $31.21 \%$ and $27.8 \%$, respectively. There was no significant aggregation of WBCs occurring in the test group compared with the control group $(P>0.05)$. The reduction in platelet aggregation to the JDBM and titanium alloys was $19.0 \%$ and $18.9 \%$, respectively. There was no significant platelet aggregation to the JDBM alloy compared with the titanium alloy $(P>0.05)$. These results indicate that blood cells suspended in the JDBM alloy did not aggregate due to blood-compatible behavior.

The initial event when a material comes in contact with blood is adsorption of proteins. Protein adsorption triggers the activation of blood cells and blood coagulation on material surfaces through a complex series of events, including the activation of platelets, leukocytes, complement, and the fibrinolytic system [19]. Therefore, the hemocompatibility of the JDBM alloy was confirmed again using a protein adsorption and platelet adhesion assay. Platelets that are altered by contacting a material may hasten thrombosis by adhering to the surface of the material and releasing or making available to the blood constituents, such as platelet factor 4. Concurrently, damaged erythrocytes may release hemoglobin and other cellular components, including thromboplastic substances and adenosine diphosphate, which is a known agonist of platelet activation. Leukocytes, when damaged, may also release materials which influence coagulation and platelets. In the present study platelets adhered to the JDBM alloy did not appear activated based on the round shape (Figure 5) compared with the platelets adherent to the titanium alloy. As shown in Figure 6, the number of platelets adherent to the JDBM and titanium alloy samples after incubation in PRP for $1 \mathrm{~h}$ had no significant change, thus indicating the very high platelet compatibility of the JDBM alloy. The results generated herein confirm the hemocompatibility behavior of the JDBM alloy, and thereby suggests that the JDBM alloy is suitable for medical applications with respect to hemocompatibility.

Collectively, the results from this study demonstrated that the JDBM alloy has excellent blood compatibility in vitro. However, there were some limitations to the current study. First, static in vitro tests may not exactly simulate the actual physiologic conditions in the human body because the human body fluid circulates dynamically. Moreover, ion concentrations differ in different parts of the human body [20]. In addition, experiments using human whole blood are needed to test the clinical applications of the JDBM alloy. Finally, although the novel JDBM alloy has excellent hemocompatibilities in vitro, further in vitro examinations and in vivo studies are necessary to validate and supplement the results of our study.

\section{Conclusion}

In the present study, the in vitro hemocompatibilities of the JDBM alloy were investigated to explore its potential as a degradable medical biomaterial. From the results of the current experiments, the JDBM alloy did not induce significant blood cell aggregation, platelet adhesion, and protein adsorption comparison with the titanium alloy (control). The results obtained from these investigations suggest that the JDBM alloy is a promising material for medical applications with respect to hemocompatibility.

This work was supported by Major Basic Research Project of Shanghai Science and Technology Commission $(11 D J 1400304,11 D J 1400301)$ and the National Natural Science Foundation of China (81071452, 81271961 and 81271998).

1 Witte F. The history of biodegradable magnesium implants: A review. Acta Biomater, 2010, 6: 1680-1692

2 Zberg B, Uggowitzer P J, Löffler J F. MgZnCa glasses without clinically observable hydrogen evolution for biodegradable implants. Nat Mater, 2009, 8: 887-891

3 Li Z J, Gu X N, Lou S Q, et al. The development of binary Mg-Ca alloys for use as biodegradable materials within bone. Biomaterials, 2008, 29: 1329-1344

$4 \mathrm{Xu} \mathrm{L} \mathrm{P,} \mathrm{Yu} \mathrm{G} \mathrm{N,} \mathrm{Zhang} \mathrm{E} \mathrm{L,} \mathrm{et} \mathrm{al.} \mathrm{In} \mathrm{vivo} \mathrm{corrosion} \mathrm{behavior} \mathrm{of}$ $\mathrm{Mg}-\mathrm{Mn}-\mathrm{Zn}$ alloy for bone implant application. J Biomed Mater Res A, 2007, 83: 703-711

5 Staiger M P, Pietak A M, Huadmai J, et al. Magnesium and its alloys as orthopedic biomaterials: A review. Biomaterials, 2006, 27: 1728-1734

6 Wang Y P, Zhu Z J, He Y H, et al. In vivo degradation behavior and biocompatibility of $\mathrm{Mg}-\mathrm{Nd}-\mathrm{Zn}-\mathrm{Zr}$ alloy at early stage. Int J Mol Med, 2012, 29: 178-184 
7 Wei J, Jia J F, Wu F, et al. Hierarchically microporous/macroporous scaffold of magnesium-calcium phosphate for bone tissue regeneration. Biomaterials, 2010, 31: 1260-1269

8 Zhang S X, Zhang X N, Zhao C L, et al. Research on an Mg-Zn alloy as a degradable biomaterial. Acta Biomater, 2010, 6: 626-640

9 Zhang S, Li J, Song Y, et al. In vitro degradation, hemolysis and MC3T3-E1 cell adhesion of biodegradable Mg-Zn alloy. Mater Sci Eng C, 2009, 29: 1907-1912

10 Wang Y P, He Y H, Zhu Z J, et al. In vitro degradation and biocompatibility of Mg-Nd-Zn-Zr alloy. Chin Sci Bull, 2012, 57: 2163-2170

11 Kirkland N T, Birbilis N, Walker J, et al. In vitro dissolution of magnesium-calcium binary alloys: Clarifying the unique role of calcium additions in bioresorbable magnesium implant alloys. J Biomed Mater Res B-Appl Biomater, 2010, 95: 91-100

12 Zhang X B, Yuan G Y, Niu J L, et al. Microstructure, mechanical properties, biocorrosion behavior, and cytotoxicity of as-extruded $\mathrm{Mg}-\mathrm{Nd}-\mathrm{Zn}-\mathrm{Zr}$ alloy with different extrusion ratios. J Mech Behav Biomed Mater, 2012, 9: 153-162

13 Wang Y P, Ouyang Y M, Peng X C, et al. Effects of degradable $\mathrm{Mg}-\mathrm{Nd}-\mathrm{Zn}-\mathrm{Zr}$ alloy on osteoblastic cell function. Int $\mathrm{J}$ Immunopath $\mathrm{Ph}, 2012,25$ : 597-606
14 Ito Y, Sisido M, Imanishi Y. Adsorption of plasma proteins and adhesion of platelets onto novel polyetherurethaneureas-relationship between denaturation of adsorbed proteins and platelet adhesion. $\mathrm{J}$ Biomed Mater Res, 1990, 24: 227-242

15 Gu X N, Zheng Y F, Cheng Y, et al. In vitro corrosion and biocompatibility of binary magnesium alloys. Biomaterials, 2009, 30: 484498

16 Johnson G, Curry B, Cahalan L, et al. In vitro assessment of blood compatibility: Residual and dynamic markers of cellular activation. J Biomater Appl, 2011, [Epub ahead of print] PMID: 22210807, doi: 10.1177/0885328211428525

17 Nilsson P H, Engberg A E, Bäck J, et al. The creation of an antithrombotic surface by apyrase immobilization. Biomaterials, 2010, 31: 4484-4491

18 Sharma C P. Blood-compatible materials: A perspective. J Biomater Appl, 2001, 15: 359-381

19 Anderson J M, Rodriguez A, Chang D T. Foreign body reaction to biomaterials. Semin Immunol, 2008, 20: 86-100

20 Lévesque J, Hermawan H, Dubé D, et al. Design of a pseudophysiological test bench specific to the development of biodegradable metallic biomaterials. Acta Biomater, 2008, 4: 284-295

Open Access This article is distributed under the terms of the Creative Commons Attribution License which permits any use, distribution, and reproduction in any medium, provided the original author(s) and source are credited. 